\title{
Simulation of Fracture of the Bone Implant with the Porous Structure
}

\author{
M. V. Korobenkov ${ }^{1, a)}$ and S. N. Kulkov ${ }^{1, b)}$ \\ ${ }^{I}$ National Research Tomsk State University, Tomsk, 634050 Russia \\ a) Corresponding author: korobenkov@ftf.tsu.ru \\ b) kulkov@ms.tsc.ru
}

\begin{abstract}
Different approaches to bone defects reconstruction with the use of ceramic materials have been developed recently. Ceramics are identical with bone matrix, provide biomedical compatibility with bone tissue and possess high strength. But with an overall high strength ceramic implants destruct in dynamic mode. The paper presents a study of the effect of the porosity gradient on the destruction of the bone implants under dynamic loading. It is shown that the fracture behavior of the bone implants is changed with increasing levels of the gradient of porosity.
\end{abstract}

\section{INTRODUCTION}

Over the past decade different approaches to bone defects reconstruction using biological materials and artificial materials including ceramics, metals, polymers and composites have been developed. Current research state analysis of osteogenesis materials shows that the main attention of researchers is focused on the materials related to the class of ceramics and ceramic composites. Ceramics are identical to the organic bone matrix by the type of chemical bond that put them in a priority position as materials for bone implants reconstruction. But despite this overall high strength the ceramic implants are destructed in dynamic mode. This work presents a study of the effects of the porosity gradient on the destruction of bone implants under dynamic loading. This research is relevant and caused by the necessity of choosing the optimal structure of the ceramics implant capable of being implanted into the bone tissue as long as possible despite the test organism loads.

\section{THE MATHEMATICAL FORMULATION. BASIC EQUATIONS}

Consider an elementary volume of a porous ceramic material under plane-wave loading. The deformation of ceramics in a condensed phase is described by means of an approach adopted in the mechanics of damaged media [1, 2]. A reference system is introduced where rectangular $O X_{1}$ and $O X_{2}$ axes lie in the model plane. The kinematics of the medium in the condensed phase is described by strain tensor and rotation tensor components. Thus we write

$$
\dot{\varepsilon}_{i j}=\frac{1}{2}\left(\frac{\partial u_{i}}{\partial x_{j}}+\frac{\partial u_{j}}{\partial x_{i}}\right), \dot{\omega}_{i j}=\frac{1}{2}\left(\frac{\partial u_{i}}{\partial x_{j}}+\frac{\partial u_{j}}{\partial x_{i}}\right) .
$$

Here $u_{i}$ is the particle-velocity vector components and $\dot{\varepsilon}_{i j}$ and $\dot{\omega}_{i j}$ are the strain and rotation rate tensor components.

In the Lagrangian reference system, the equations of conservation of mass, momentum and energy are of the form

$$
\frac{\partial \sigma_{i j}}{\partial x_{j}}=\rho \frac{\mathrm{d} u_{i}}{\mathrm{~d} t}, \frac{\mathrm{d} \rho}{\mathrm{d} t}=\rho \frac{\partial u_{i}}{\partial x_{i}}, \rho \frac{\mathrm{d} E}{\mathrm{~d} t}=\sigma_{i j} \dot{\varepsilon}_{i j},
$$

where $\sigma_{i j}$ is the stress tensor components, $\rho$ is the mass density, and $E$ is the specific internal energy per unit mass.

\footnotetext{
Physics of Cancer: Interdisciplinary Problems and Clinical Applications (PC'16)

AIP Conf. Proc. 1760, 020033-1-020033-4; doi: 10.1063/1.4960252

Published by AIP Publishing. 978-0-7354-1418-1/\$30.00
} 
The stress tensor components are written as $\sigma_{i j}=-p \delta_{i j}+S_{i j}$, where $p$ is the pressure, $\delta_{i j}$ is the Kronecker delta, and $S_{i j}$ is the stress deviator tensor components.

The pressure up to $10 \mathrm{GPa}$ is calculated by the equation of states $p=K_{1} \theta+K_{2} \theta^{2}+K_{3} \theta^{3}+\Gamma \rho_{0} E$ for compression $(\theta>0), p=K_{1} \theta$ for tension $(\theta \leq 0)$, where $K_{1}, K_{2}$ and $K_{3}$ are material constants, $\theta=\rho / \rho_{0}-1$, and $\Gamma$ is the Gruneisen coefficient.

Brittle porous ceramics in the condensed phase undergoing deformation may exhibit shear-microcrack nucleation [3-5]. As this takes place, the shear strength of the elementary volume of the material containing cracks decreases. To account for loss in the local shear strength of the elementary volume, we have introduced the damage parameter $D$ for the medium

$$
D=\sum_{k=0}\left(\frac{\Delta \varepsilon^{\mathrm{p}}}{\varepsilon_{\mathrm{f}}}\right)_{k},
$$

where $\left(\Delta \varepsilon^{\mathrm{p}}\right)_{k}=\int_{t_{k}}^{t_{k+1}} \dot{\varepsilon}_{u}^{\mathrm{p}} \mathrm{d} t, \dot{\varepsilon}_{u}^{\mathrm{p}} \mathrm{d} t$ is the equivalent inelastic strain rate tensor, and $\varepsilon_{\mathrm{f}}$ is the strain for which the elementary volume is fragmented due to the cracking effect.

In the case of no cracks in the condensed phase in the initial state, the damage parameter is zero. The local failure criterion for the condensed phase is $D=1$. The effective stress deviator tensor in a damaged medium is given by the relation $S_{i j}=(1-D)\left[S_{i j}\right]_{\mathrm{c}}$, where $\left[S_{i j}\right]_{\mathrm{c}}$ is the stress tensor components in the condensed phase.

Within the model put forward in [1], the ultimate equivalent shear strain at fracture, $\varepsilon_{\mathrm{f}}$ is approximated as $\varepsilon_{\mathrm{f}}=D_{1}\left(P^{*}+T^{*}\right)^{D_{2}}$, where $T^{*}=\left(T-T_{\mathrm{r}}\right) /\left(T_{\mathrm{m}}-T_{\mathrm{r}}\right), T_{\mathrm{m}}$ is the melting temperature, $T_{\mathrm{r}}=293 \mathrm{~K}, P^{*}=\rho / P_{\mathrm{HEL}}, P_{\mathrm{HEL}}$ is the pressure corresponding to the Hugoniot elastic limit, and $D_{1}$ and $D_{2}$ are material constants.

The stress deviator tensor is calculated in the framework of the Drucker-Prager model with the JohnsonHolmquist plastic potential [1]. Thus we get

$$
\frac{\mathrm{d}\left[S_{i j}\right]_{\mathrm{c}}}{\mathrm{d} t}=2 \mu\left(\dot{\varepsilon}_{i j}^{\mathrm{e}}-\frac{1}{3} \dot{\varepsilon}_{k k}^{\mathrm{e}} \delta_{i j}\right), \frac{\mathrm{d}\left[S_{i j}\right]_{\mathrm{c}}}{\mathrm{d} t}=\left[\dot{S}_{i j}\right]_{\mathrm{c}}-\left[S_{i k}\right]_{\mathrm{c}} \dot{\omega}_{j k}-\left[S_{j k}\right]_{\mathrm{c}} \dot{\omega}_{i k}
$$

Here $\mu$ is the shear modulus for the condensed phase, and

$$
\begin{gathered}
\dot{\varepsilon}_{i j}^{\mathrm{e}}=\dot{\varepsilon}_{i j}-\dot{\varepsilon}_{i j}^{\mathrm{p}}, \dot{\varepsilon}_{i j}^{\mathrm{p}}=\dot{\varphi} \frac{\partial g}{\partial \sigma_{i j}}, g=\left[\frac{1}{2} S_{i j} S_{i j}\right]^{1 / 2}-\sigma_{\mathrm{s}}, \\
\sigma_{\mathrm{s}}=A\left(P^{*}+T^{*}\right)^{m}(1+C \ln \dot{\varepsilon}),
\end{gathered}
$$

where $A, C$, and $m$ are material constants, $\dot{\varepsilon}=\dot{\varepsilon}_{\mathrm{u}} / 1\left[C^{-1}\right]$ is the normalized equivalent strain rate tensor, and $\dot{\varphi}$ is a coefficient.

As the material undergoes inelastic deformation, the damage is assumed to accumulate, which degrades the overall load carrying capacity of the materials. The Johnson-Holmquist plastic potential was developed for the simulation of the compressive and shear induced strength and failure of brittle materials. Damage is accumulated as a ratio of incremental plastic strain is pressure depends as described below.

There are two methods for the damage application to the material strength. The default gradual failure type results in damage being incrementally applied to the material strength as it accumulates. If the instantaneous failure type is selected, damage accumulates over time; however, it is only applied to the failure surface when its value reaches unity. The materials strength instantaneously transitions from impact to complete failure in this case. The Johnson-Holmquist plastic potential includes an option to represent volumetric dilation of the material due to shear deformation (bulking). The work done in deforming the material inelastically in shear can be converted into a pressure increase, hence volumetric dilation (if unconstrained). The amount of work which is converted into dilation pressure is controlled through the bulking constant. This can have values ranging from 0 (representing no shear induced dilatancy) to 1 (producing maximum dilatancy effects).

Erosion control was chosen for visualization of the failure. Erosion is a numerical mechanism for the automatic removal of elements during a simulation. The primary reason for using erosion is to remove very discorted elements from a simulation before the elements become inverted. This ensures that the stability timestep remains at a reasonable level and solution can continue to the desired termination time. The geometrical strain was chosen as a type of erosion. Geometrical strain is a measure of the distortion of an element. 

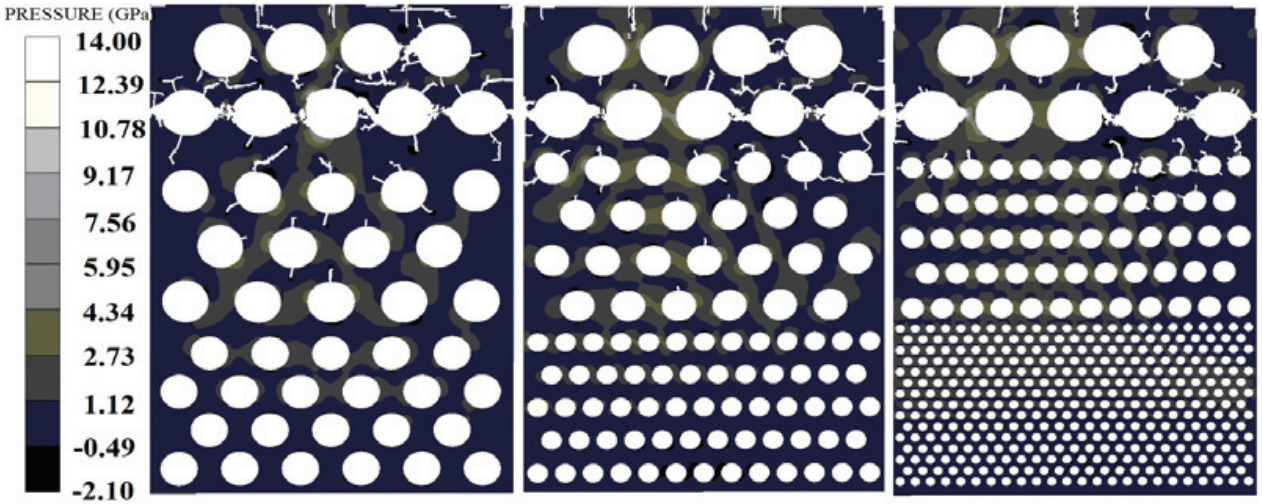

FIGURE 1. The pressure field for the three levels of pore gradient structure of $\mathrm{ZrO}_{2}$ ceramics with amplitude of $2.5 \mathrm{GPa}$

For instance, in $\mathrm{ZrO}_{2}$ in the condensed phase, the following values of the coefficients were used: $\rho_{0}=6.05 \mathrm{~g} / \mathrm{cm}$, $K_{1}=212 \mathrm{GPa}, K_{2}=0, K_{3}=0, \Gamma=1, \mu=85 \mathrm{GPa}, D_{1}=0.01, D_{2}=0.7, P_{\mathrm{HEL}}=65.7 \mathrm{GPa}, A=57.8 \mathrm{GPa}, C=0.007$, $m=0.6$.

\section{SIMULATION RESULTS}

The models of ceramic implants with porous structure were generated. Figure 1 shows 3 structures with different porous gradient levels $(20 \%, 40 \%$ and $60 \%)$, and total porosity is $30 \%$ for all the layers. In this model the porosity gradient means that the size of pores in subsequent layer is different from the previous one by the value of the gradient of porosity. The dimensions of computational domain are $300 \times 450 \mu \mathrm{m}$. The size of the pores in the first layer is $24 \mu \mathrm{m}$. The loading of the computational domain is given by applying speed to the upper boundary face. On the side boundaries are set translational boundary conditions.

Figure 1 shows the pressure field for the three levels of gradient structure of $\mathrm{ZrO}_{2}$ ceramics with amplitude of $2.5 \mathrm{GPa}$ and time moment $100 \mathrm{~ns}$. The pores are the cause of inhomogeneous stress field. The maximum compression pressure is reached at a higher level of porous gradient. The destruction of ceramics occurs in a region of tensile stresses and the pressure level does not exceed $2 \mathrm{GPa}$.

Figure 2 shows the evolution of stress field and total energy at the consecutive point of time 20 and 40 ns. The stepped type of the graph can be explained as the dissipation of the energy associated with the destruction of ceramics.

Figure 3 shows a graph of the character of deformation work influence on bone ceramic implants with the porosity gradient level. Implants with lower porosity gradient are more vulnerable to destruction. The fracture pattern is associated with the formation of trunk cracks in a big porous area.
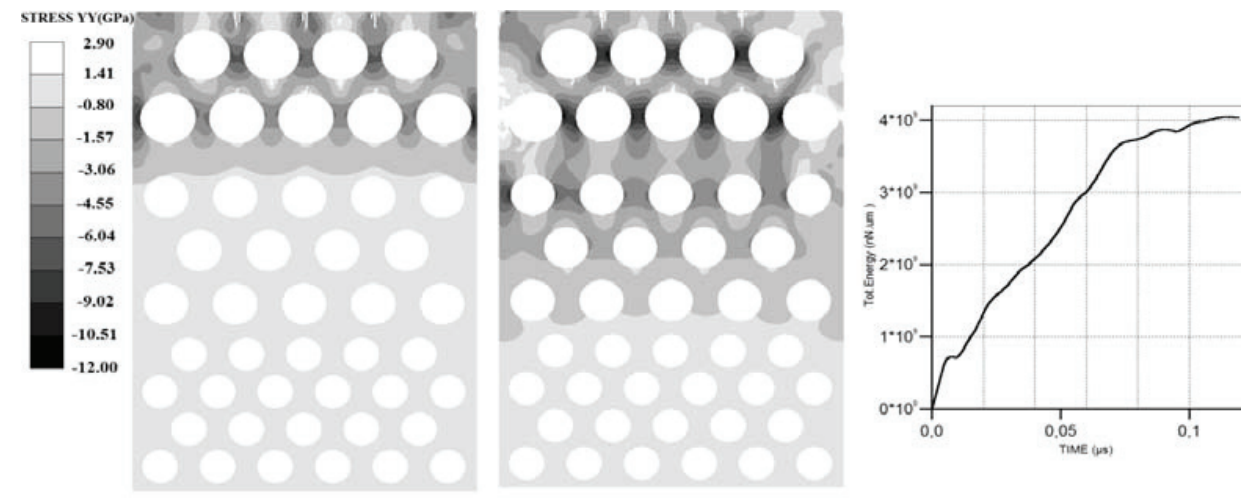

FIGURE 2. The evolution of stress field in a shock wave 


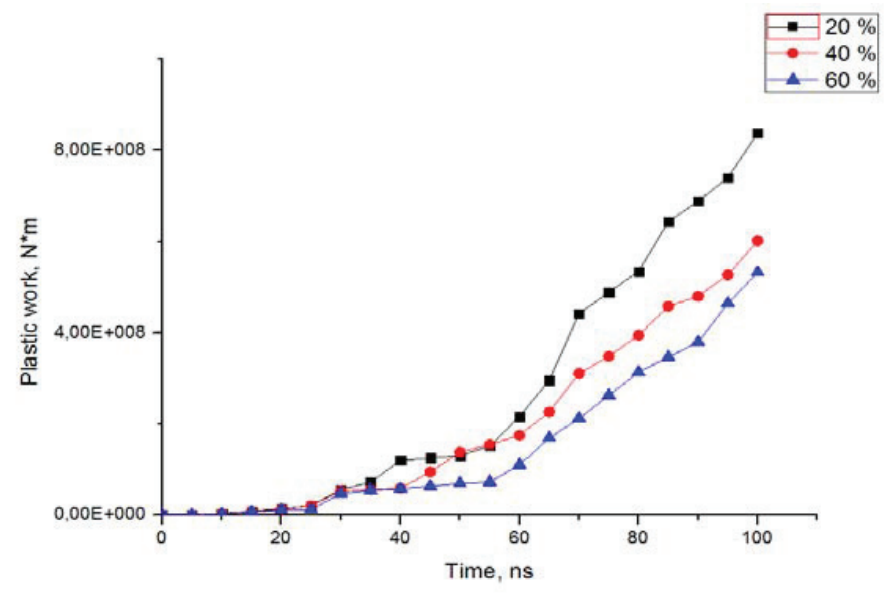

FIGURE 3. The result of the work of plastic deformation in ceramic

In this case of elastic-brittle behavior of bone ceramic implants, the size of J-integral corresponding to the work of plastic deformation is spent on the cracks opening. In this case $J=J_{1 \mathrm{c}}=W_{\text {pd }}$, where the $J_{1 \mathrm{c}}$ is fracture toughness, $W_{\mathrm{pd}}$ is the plastic deformation work.

\section{CONCLUSION}

Here is shown that the presence of pores with a gradient structure in brittle ceramic implant makes failure wave generation difficult under dynamic loadings. Pore structure has a profound impact on the strength of brittle $\mathrm{ZrO}_{2}$ ceramic implants. The dynamic durability of the implant is achieved by increasing in the level of pore gradient structure. The fracture pattern is associated with the formation of trunk cracks in the area with the big porous.

\section{ACKNOWLEDGMENTS}

The work was performed within the implementation of Tomsk State University Competitiveness Improvement Program and with partial financial support of the Ministry of Education and Science of the Russian Federation within State task No. 16.2004.2014/K and was funded by RFBR, according to the research project No. 16-31-60059 mol_a_dk.

The study reported in this article was conducted according to accepted ethical guidelines involving research in humans and/or animals and was approved by an appropriate institution or national research organization. The study is compliant with the ethical standards as currently outlined in the Declaration of Helsinki. All individual participants discussed in this study, or for whom any identifying information or image has been presented, have freely given their informed written consent for such information and/or image to be included in the published article.

\section{REFERENCES}

1. G. R. Johnson and T. J. Holmquist, An improved computational constitutive model for brittle materials, in High-Pressure Science And Technology, AIP Conference Proceedings 309, edited by S. C. Schmidt, et al. (American Institute of Physics, Colorado Springs, Colorado, 1993), p. 981, http://dx.doi.org/10.1063/1.46199.

2. V. A. Skripnyak, E. G. Skripnyak, and T. V. Zhukova, Chem. Phys. 21(9), 76-82 (2002).

3. G. I. Kanel, A. A. Bogach, S. V. Rzorenov, et al, A Study of the failure wave phenomenon in brittle materials, in Shock Compression of Condensed Matter-2003, AIP Conference Proceedings 706, edited by M. D. Furnish, et al. (American Institute of Physics, Portland, Oregon, 2003), p. 739, http://dx.doi.org/10.1063/1.1780344.

4. R. L. Woodward, W. A. Gooch, R. G. O’Donnel, et al., Int. J. Impact Engng. 15(5), 605-618 (1994).

5. D. E. Grady, Mech. Matter 29, 181-203 (1988). 